\title{
Shrunken2 Sweet Corn Yield and the Chemical Components of Quality
}

\author{
Adamson D. Wong', John A. Juvik², David C. Breeden'1, and John M. Swiader ${ }^{3}$ \\ Department of Horticulture, University of Illinois, Urbana, IL 61801
}

Additional index words. Zea mays L., sugars, dimethyl sulfide, endosperm mutations, shrunken2

\begin{abstract}
Extensive variability was found among 24 currently available commercial sh2 hybrids of sweet corn (Zea mays L.) for yield and yield components, and for the chemical components of eating quality. The primary source of variation was explained by genotypic differences, with the environmental effects due to planting locations having a minor influence. Kernel sugar concentrations, however, had a highly significant level of genotype by environment interaction. The extensive genotypic variability among the $s h 2$ hybrids indicated that allelic variation at other loci is profoundly influencing sucrose and total sugar levels in freshly harvested sweet corn. In each case, the kernel chemical components of quality decreased from 20 to 29 days after pollination (DAP). Mean performance of $s h 2$ hybrids for yield, yield components, and kernel quality parameters was in all cases equal or better than the hybrids homozygous for the sul endosperm mutation. In addition, there were no strong negative relationships between yield and some of the important chemical components of kernel quality, suggesting that it may be feasible to develop superior $s h 2$ hybrids with acceptable yield potential and improved eating quality targeted for the different sweet corn markets.
\end{abstract}

The primary components of sweet corn eating quality associated with consumer preference are kernel flavor, texture, and aroma (Flora and Wiley, 1974). Sweetness in sweet corn constitutes most of what the average consumer perceives as flavor (Culpepper and Magoon, 1927), and it is closely related to kernel sucrose content (Reyes et al., 1982), the primary sugar in developing kernels (Cobb and Hannah, 1981). Textural eating quality of sweet corn consists of several factors, including pericarp tenderness (Bailey and Bailey, 1938), level of water soluble polysaccharides or phytoglycogen (Culpepper and Magoon, 1927), and moisture content (Wann et al., 1971). Aroma, which has not been as easily defined as either sweetness or texture, is most often associated with dimethyl sulfide (DMS), a volatile compound described by taste panelists as lending a pleasing and "corn-like" character (Wiley, 1985). Several studies have reported significant differences in DMS levels among sweet corn genotypes and harvest maturities, with DMS content decreasing with increasing kernel age (Dignan and Wiley, 1976; Williams and Nelson, 1973). Traditional sweet corn hybrids homozygous for the sugaryl (sul) mutation are characterized by rapid moisture loss and the conversion of endosperm sugars to starch. This decline in quality places a time constraint on the shipment of fresh sweet corn to major urban markets and also creates a very narrow harvest window for the sweet corn processing industry (Marshall, 1987). Consequently, there has been a dramatic shift away from the traditional sul hybrids to hybrids with the shrunken2 (sh2) mutation. Compared to $s u l$, the $s h 2$ phenotype is characterized by kernels that have two to three times more sucrose at harvest maturity (Creech, 1965), can retain higher sugar and moisture content for longer postharvest periods (Garwood et al., 1976), and is preferred by consumers in taste tests (Evensen and Boyer, 1986; Showalter and Miller, 1962).

While the long-distance shipping market of fresh sweet corn

Received for publication 20 May 1993. Accepted for publication 8 Nov. 1993. This work was supported in part by Knorr Foods Company, Ltd. of Japan and by grant number US-1709-89 from the US-Israel Binational Agricultural Research and Development Fund (JAJ). The cost of publishing this paper was defrayed in part by the payment of page charges. Under postal regulations, this paper therefore must be hereby marked advertisement solely to indicate this fact.

${ }^{\mathrm{I}}$ Graduate research assistants.

${ }^{2}$ Associate professor, to whom reprint requests should be addressed.

${ }^{3}$ Associate professor. has largely converted to the use of $s h 2$ hybrids, acceptance and use in the processing sector of the industry has lagged behind because there has been a perception that these hybrids display poor stand establishment and yield potential. Some of the first sh2 hybrids released in the 1960s and 1970s suffered from these problems, but numerous extension publications suggest that more recent hybrids have improved yield and enhanced quality. Comparison of $s h 2$ hybrids with widely used sul hybrids for yield, yield components, and kernel quality is needed to determine any improvements. The following experiments were designed to provide information on the phenotypic variability among commercial $s h 2$ hybrids for yield and yield components, and for the chemical composition of eating quality. Additional objectives were to determine the effect of harvest maturity on the chemical composition of eating quality, examine the relationships between yield, yield components, and quality attributes, and provide sweet corn breeders with the information needed to develop superior $s h 2$ hybrids.

\section{Materials and Methods}

Field design. In 1989, 31 commercial hybrids, consisting of 24 $s h 2$, five sul, and two sugary enhancer (se1) types, were grown in a randomized complete-block design with four replications on a Flanagan silt loam (fine, montmorillonitic, mesic Aquic Argiudoll), characterized by high $(5 \%)$ organic matter content and high nutrient holding capacity (a CEC of $24.0 \mathrm{meq} \cdot 100 \mathrm{~g}^{-1}$ soil), at the South Urbana Research Farm of the University of Illinois. The 24 sh 2 hybrids were chosen for their potential use by the processing industry, while the five sul types were selected as standards currently used in commercial sweet corn production. Each plot consisted of four rows spaced $90 \mathrm{~cm}$ apart, with each row containing 30 hills at $25-\mathrm{cm}$ intervals. Plots were established by hand planting to result in an approximate stand of 50,000 plants/ha. Plants in the two outer rows were self-pollinated.

The following year, six sh 2 hybrids, which varied in yield and kernel chemical composition in the 1989 study, were planted on a Plainfield sand (mixed, mesic Typic Udipsamment) at the Illinois River Valley Sand Field (IRVSF) in Kilbourne, Illinois. This site, with soil organic matter content of $0.7 \%$ and a CEC of $2.5 \mathrm{meq} \cdot 100$ $\mathrm{g}^{-1}$, was selected to determine the effect of environment on yield and chemical composition of quality in sh2 sweet corn. Four replicates of the six genotypes were planted in a randomized 
complete-block design at a planting density equivalent to the 1989 field experiment. Supplemental sprinkler irrigation was provided as needed throughout the growing season.

Yield and yield component evaluations. Pollination of the central row(s) was permitted to occur via open pollination. From 20 to 25 ears in the outer rows were manually self-pollinated at plot mid-silk for subsequent sample harvest. Husked ears from the central row(s) were used for comparison of ear uniformity, the components of yield (i.e., ear weight, ear length, and kernel depth), and estimates of yield (i.e., potential ear yield and kernel yield). Open-pollinated ears were harvested 23 days after the plot mid-silk date and evaluated within $24 \mathrm{~h}$.

A rating scale of 0 to 4 was used to rate husked ear uniformity. Genotypes were assigned a uniformity rating of 0 when less than $10 \%$ of the ears harvested in a plot had good marketable quality, up to a rating of 4 when more than $90 \%$ of the ears harvested were identical in size, shape, and overall appearance. Ten of the ears harvested from each plot were then selected randomly and measured to determine average ear weight $(\mathrm{g})$ and average ear length $(\mathrm{cm})$. Mean kernel depth $(\mathrm{cm})$ was estimated from averaged measurements from three of these ears. Ears were broken in half and kernel depth was measured with a ruler.

Potential yield was the estimated weight in MT.ha ${ }^{-1}$ of husked ears, assuming a stand density of 45,000 plants/ha. Kernel yield was estimated by using the following formula: kernel yield $=\left[\pi\left(\mathrm{r}_{\text {ear }}\right)^{2} 1_{\text {ear }}\right]-\left[\pi\left(\mathrm{r}_{\text {cob }}\right)^{2} 1_{\text {ear }}\right] / \pi\left(\mathrm{r}_{\text {ear }}\right)^{2} 1_{\text {ear }} \times$ potential yield, where ${ }^{\mathrm{r}}$ ear $=$ ear radius in $\mathrm{cm}$, ${ }^{1}$ ear $=$ ear length in centimeters, and $r_{\text {cob }}=$ ear radius - kernel depth in centimeters. The first part of the formula is essentially the proportion of kernel volume in an average ear. Multiplying this proportion by the potential yield would give an estimate of kernel yield in $\mathrm{MT} \cdot \mathrm{ha}^{-1}$ that the various genotypes would provide the processors after separation of the kernels from the husks by commercial cutting machines.

Chemical analysis. All ears harvested for chemical analyses were hand-pollinated to ensure uniform maturity and genetic purity. As many plants as possible within a plot were pollinated on the same day with a minimum of about ten plants in each of the outer rows. Harvests of self-pollinated ears in each plot were made at 3-day intervals from 20 to 29 days after pollination (DAP) in 1989 , and from 20 to 26 DAP in 1990. These stages were chosen because sugars reach maximum levels at $\approx 21$ DAP and sweet corn typically is harvested at this stage of maturity (Carey et al., 1984). No ears were harvested within $1 \mathrm{~m}$ of the beginning and end of each row to avoid edge effects on yield and chemical analyses.

At each harvest date, four self-pollinated ears were picked at random for each genotype in each of the four replicates. Directly after harvest, ears were husked and silk was removed. To reduce sample storage volume, a portion of the tip and butt of each ear was discarded. The remaining portion was immediately frozen in liquid nitrogen to stop all metabolic activity. Frozen ears were placed in labeled plastic bags, placed on dry ice, and transported to cold storage at about $-20 \mathrm{C}$. The time from harvest to freezing in liquid nitrogen was generally $<15 \mathrm{~min}$. By resuspension of the frozen ears in liquid nitrogen, a central band of kernels $(\approx 2.5 \mathrm{~cm})$ was later removed from individual ears with a screwdriver. Kernels from each of the four ear samples were bulked. Subsamples were then weighed, freeze-dried, weighed again for moisture content determination, ground into powder with a coffee grinder, and stored in the freezer at $-20 \mathrm{C}$ for subsequent sugar and DMS analyses.

Sugars and DMS levels were analyzed using a gas chromatograph (model 5790A; Hewlett-Packard, Palo Alto, Calif.) with an autosampler (model 7671A; Hewlett-Packard), a 12-m capillary column (model Ultra-l; Hewlett-Packard), a flame-ionization de- tector, and an integrator (model 3390A; Hewlett-Packard). Helium was used as the carrier gas.

Determination of fructose, glucose, and sucrose concentrations (mg. $\mathrm{g}^{-1}$ dry weight of seed) was conducted as described by Juvik and LaBonte (1988), who reported this procedure effective in extracting $>98 \%$ of the sugars in ground kernel samples. Total sugars were obtained by adding the amounts of fructose, glucose, and sucrose. Dimethyl sulfide level was analyzed using the procedure of Breeden and Juvik (1992), and expressed as $\mu \mathrm{g} \cdot \mathrm{g}^{-1}$ dry weight of seed. This method avoids the problems of headspace gas analysis by keeping the DMS in solution, and is compatible with automated multiple-sample gas chromatographic analysis.

Data evaluation. Analysis of variance (ANOVA) was performed on yield and yield components for each location. A factorial design was used to analyze for kernel moisture content, sugar concentration, and DMS level, with genotype and kernel maturity as main factors. Least significant difference (LSD) values calculated at $P=0.05$ (Steel and Torrie, 1960) were used to compare hybrid means within kernel maturity, and kernel maturities within hybrids. Based on the ANOVA procedure, the percentage of variability explained by each source of variation was computed for yield and yield components at $23 \mathrm{DAP}$, and for kernel chemical composition at 20 DAP for the six sh 2 hybrids used in both locations. These were calculated by dividing the sum of squares of the factor involved by the total sum of squares and expressed as percentages.

To address the issue of $s h 2$ yield, yield components, and kernel quality performance, replicate means averaged over the $24 \operatorname{sh} 2$ hybrids in the Urbana planting were compared with replicate means averaged over the five widely used sul hybrids using Student's $t$ test as described by Steel and Torrie (1960). Yield and yield component evaluations were tested from the 23 DAP harvest, while kernel chemical compositions were computed from the 20 DAP harvest. The two sel hybrids were not included in this evaluation, since the sample size was insufficient. In addition, simple phenotypic correlations over all the sh 2 hybrids grown at the South Urbana Research Farm were determined between yield, yield component evaluations, and kernel chemical compositions at 23 DAP, and between chemical factors of quality at 20 and 23 DAP.

\section{Results and Discussion}

Yield and yield component evaluations. In 1989, significant differences among genotypes were observed for all of the yield and yield component parameters measured (Table 1). Ear uniformity, an important characteristic for the fresh market industry, ranged from a low of $1.0 \mathrm{in}$ 'Style Sweet' and 'Sweetie 70' to a high of 3.5 in 'Sweetie 82'. Kernel depth and kernel yield, two closely related parameters important to the processing industry, were generally highest in 'Florida Staysweet' and 'Sweetie 82', and lowest in 'Sweetie 70'. Ear weight also varied significantly, with highest values in 'FMX 261' and 'Summer Sweet 7210'. Among the sh2 hybrids, potential yields showed an almost 2-fold difference, ranging from 6.6 MT'ha- ${ }^{-1}$ in 'Sweetie 70' and 7.2 MT'ha-1 in 'Crisp-N-Sweet 620' to $12.5 \mathrm{MT} \cdot \mathrm{ha}^{-1}$ in 'Summer Sweet 7210 '.

Similar to the response in 1989, significant differences were observed in 1990 among the six sh2 hybrids for each of the yield variables evaluated (Table 1). Ear weight and ear length at the IRVSF site were generally highest in 'Crisp-N-Sweet 710' and 'FMX 263', and along with good kernel depth provided for high potential yields and kernel yields. 'Summer Sweet 7210' produced a generally smaller ear in 1990, with lower potential yield and kernel yield. 'Sweetie 70' at the IRVSF station produced small ears 
Table 1. Yield and yield component evaluations of hybrids harvested at 23 DAP at South Urbana Research Farm (1989) and the Illinois River Valley Sand Field (1990).

\begin{tabular}{|c|c|c|c|c|c|c|}
\hline \multirow[b]{2}{*}{ Hybrid $^{\mathrm{z}}$} & \multirow{2}{*}{$\begin{array}{c}\text { Ear } \\
\text { uniformity } \\
(0 \text { to } 4)^{\mathrm{x}}\end{array}$} & \multirow{2}{*}{$\begin{array}{l}\text { Ear } \\
w t \\
(\mathrm{~g})\end{array}$} & $\begin{array}{c}\text { Ear } \\
\text { length }\end{array}$ & $\begin{array}{c}\text { Kernel } \\
\text { depth }\end{array}$ & \multirow{2}{*}{$\begin{array}{c}\begin{array}{c}\text { Potential } \\
\text { yield }\end{array} \\
\end{array}$} & \multirow{2}{*}{$\begin{array}{c}\begin{array}{c}\text { Kernel } \\
\text { yield }\end{array} \\
\frac{1}{1} \text { ) } \\
\end{array}$} \\
\hline & & & \multicolumn{2}{|c|}{$(\mathrm{cm})$} & & \\
\hline \multicolumn{7}{|c|}{1989} \\
\hline Bunker Hill & 2.1 & 210.2 & 18.9 & 0.75 & 9.3 & 5.2 \\
\hline Crisp-N-Sweet 620 & 1.5 & 163.2 & 17.4 & 0.77 & 7.2 & 4.3 \\
\hline Crisp-N-Sweet 710 & 2.5 & 231.8 & 20.6 & 0.72 & 10.3 & 5.4 \\
\hline Excel GSS3724 & 2.4 & 191.7 & 20.6 & 0.72 & 8.5 & 4.8 \\
\hline Florida Staysweet & 2.6 & 238.6 & 20.4 & 0.94 & 10.6 & 6.8 \\
\hline FMX 263 & 2.6 & 235.2 & 21.0 & 0.82 & 10.5 & 6.1 \\
\hline Illini Gold & 3.1 & 238.6 & 21.0 & 0.79 & 10.6 & 6.1 \\
\hline Landmark & 2.2 & 254.5 & 20.9 & 0.86 & 11.3 & 6.7 \\
\hline Northern Extrasweet & 1.4 & 171.2 & 18.5 & 0.66 & 7.6 & 4.0 \\
\hline Pinnacle & 2.6 & 223.9 & 20.8 & 0.79 & 10.0 & 5.7 \\
\hline SCH 4006 & 1.9 & 239.8 & 20.9 & 0.79 & 10.7 & 6.1 \\
\hline SCH 4415 & 3.2 & 250.0 & 19.2 & 0.84 & 11.1 & 6.4 \\
\hline Style Sweet & 1.0 & 198.7 & 17.3 & 0.78 & 8.8 & 5.2 \\
\hline Sucro & 2.9 & 250.0 & 20.8 & 0.84 & 11.1 & 6.5 \\
\hline Summer Sweet 7210 & 2.6 & 280.3 & 21.0 & 0.77 & 12.5 & 6.6 \\
\hline Summer Sweet 8000 & 3.1 & 246.6 & 19.9 & 0.85 & 11.0 & 6.4 \\
\hline Supersweet Jubilee & 2.5 & 200.8 & 19.6 & 0.80 & 8.9 & 5.1 \\
\hline Sweet Belle & 2.8 & 242.0 & 21.0 & 0.81 & 10.8 & 5.6 \\
\hline Sweetie 70 & 1.0 & 149.5 & 18.8 & 0.56 & 6.6 & 3.2 \\
\hline Sweetie 76 & 2.4 & 234.1 & 18.4 & 0.86 & 10.4 & 5.6 \\
\hline Sweetie 82 & 3.5 & 260.2 & 20.0 & 0.90 & 11.6 & 6.9 \\
\hline Upmost & 1.9 & 210.2 & 19.1 & 0.79 & 9.4 & 5.4 \\
\hline Wisc. Natl. Sweet 9000 & 2.5 & 228.3 & 20.4 & 0.82 & 10.2 & 5.9 \\
\hline Xtra Sweet 82 & 1.6 & 244.2 & 20.0 & 0.83 & 10.9 & 6.2 \\
\hline Bellringer $s u l$ & 2.8 & 212.5 & 19.6 & 0.86 & 9.4 & 5.9 \\
\hline FMX 261 sul & 2.5 & 284.1 & 21.6 & 0.80 & 12.6 & 6.7 \\
\hline Jubilee sul & 3.0 & 234.1 & 20.8 & 0.83 & 10.4 & 6.2 \\
\hline Seneca Horizon sul & 1.9 & 230.7 & 16.3 & 0.78 & 10.3 & 5.4 \\
\hline Style Pack sul & 2.4 & 269.3 & 20.3 & 0.80 & 12.0 & 6.6 \\
\hline Maple Sweet se1 & 1.5 & 143.2 & 16.3 & 0.72 & 6.4 & 3.6 \\
\hline Merlin Supersweet se1 & 1.2 & 156.8 & 17.8 & 0.69 & 7.0 & 3.8 \\
\hline $\operatorname{LSD}(P=0.05)^{\mathrm{y}}$ & 1.1 & 39.3 & 1.6 & 0.1 & 1.8 & 1.2 \\
\hline \multicolumn{7}{|c|}{1990} \\
\hline Crisp-N-Sweet 710 & 3.1 & 235.6 & 20.4 & 1.20 & 10.5 & 7.6 \\
\hline FMX 263 & 2.7 & 232.4 & 21.1 & 1.18 & 10.3 & 7.5 \\
\hline Summer Sweet 7210 & 3.0 & 211.0 & 20.0 & 1.17 & 9.4 & 6.8 \\
\hline Supersweet Jubilee & 3.0 & 199.0 & 19.6 & 1.22 & 8.8 & 6.7 \\
\hline Sweetie 70 & 2.1 & 190.5 & 19.0 & 0.92 & 8.5 & 5.3 \\
\hline Sweetie 82 & 3.5 & 218.8 & 18.7 & 1.23 & 9.7 & 7.2 \\
\hline $\operatorname{LSD}(P=0.05)^{\mathrm{y}}$ & 0.4 & 28.6 & 0.8 & 0.1 & 1.3 & 1.0 \\
\hline
\end{tabular}

${ }_{\mathrm{z}} \mathrm{s} 2$ endosperm mutation unless otheruise indicated.

$\mathrm{y}_{\text {LSD }}$ between hybrid means at $P=0.05$.

${ }^{\mathrm{x}}$ Rating of 0 was assigned when $<10 \%$ of the ears harvested in a plot had good marketable quality up to a rating of 4 when $>90 \%$ of the ears harvested were identical in size, shape, and overall appearance.

and low yield, while 'Sweetie 82' maintained a uniform ear with large kernels, resulting in high kernel yield. With the exception of 'Sweetie 70', which performed marginally at both sites, differences in the relative ranking of genotypes between locations for ear weight, potential yield, and kernel yield indicated that there was significant genotype $\times$ environment interaction influencing hybrid yield and yield component parameters.

Chemical components of kernel quality. Significant differences among hybrids and over harvest dates were observed in each of the kernel chemical variables for the 31 hybrids grown in Urbana (Table 2). At 20 DAP, kernel moisture content in sh2 hybrids ranged from $73.1 \%$ in 'Crisp-N-Sweet 620 ' to $76.8 \%$ in 'Florida Staysweet', suggesting that the hybrids varied in their rates of ear maturation. As anticipated, kernel moisture content decreased with increasing harvest maturity, dropping 5.2\% from 20 to 29 DAP, or $0.6 \% /$ day among the $s h 2$ hybrids.

Variation in the concentrations of individual and total sugars was significant among the sh2 hybrids during the 1989 season (Table 2, refer to the bottom of table for LSDs). Except in three of the $s h 2$ hybrids, sucrose was the primary sugar, accounting for $76.7 \%$ of the mean total sugar content. Of the $24 s h 2$ hybrids evaluated, 'Upmost', 'Wisconsin Natural Sweet 9000', and 'Xtra 
Table 2. Kernel composition for hybrids grown at South Urbana Research Farm.

\begin{tabular}{|c|c|c|c|c|c|c|c|}
\hline \multirow[b]{2}{*}{ Hybrid $^{z}$} & \multirow[b]{2}{*}{$\mathrm{DAP}^{\mathrm{y}}$} & \multirow{2}{*}{$\begin{array}{c}\text { Moisture } \\
(\%)\end{array}$} & Fructose & Glucose & Sucrose & $\begin{array}{c}\text { Total } \\
\text { sugars }\end{array}$ & \multirow{2}{*}{$\begin{array}{c}\text { DMS } \\
\left(\mu \mathrm{g} \cdot \mathrm{g}^{-1}\right)\end{array}$} \\
\hline & & & \multicolumn{4}{|c|}{$\left(\mathrm{mg} \cdot \mathrm{g}^{-1}\right)$} & \\
\hline \multirow[t]{4}{*}{ Bunker Hill } & 20 & 74.8 & 22.8 & 25.8 & 261.5 & 310.1 & 77.5 \\
\hline & 23 & 73.2 & 21.6 & 26.8 & 258.6 & 306.9 & 41.8 \\
\hline & 26 & 71.8 & 18.9 & 22.3 & 206.1 & 247.4 & 35.0 \\
\hline & & 1.7 & 6.8 & 7.6 & 19.7 & 22.0 & 14.8 \\
\hline \multirow[t]{4}{*}{ Crisp-N-Sweet 620} & 20 & 73.1 & 40.7 & 43.6 & 371.0 & 455.2 & 67.4 \\
\hline & 23 & 71.3 & 40.1 & 42.8 & 320.7 & 403.6 & 50.2 \\
\hline & 26 & 69.2 & 26.8 & 29.7 & 260.7 & 317.3 & 28.8 \\
\hline & & 0.8 & 7.7 & 7.6 & 43.9 & 40.7 & 14.8 \\
\hline \multirow[t]{4}{*}{ Crisp-N-Sweet 710} & 20 & 75.8 & 41.5 & 48.6 & 317.5 & 407.6 & 184.6 \\
\hline & 23 & 73.3 & 36.3 & 42.5 & 286.9 & 365.7 & 107.3 \\
\hline & 26 & 72.0 & 29.6 & 35.2 & 228.3 & 293.1 & 59.4 \\
\hline & & 0.5 & 8.1 & 6.9 & 40.2 & 44.9 & 33.3 \\
\hline \multirow[t]{4}{*}{ Excel GSS3724 } & 20 & 73.2 & 15.3 & 16.4 & 115.3 & 147.1 & 72.2 \\
\hline & 23 & 72.7 & 14.4 & 15.8 & 109.6 & 139.9 & 39.3 \\
\hline & 26 & 71.8 & 13.3 & 14.5 & 106.1 & 133.9 & 24.3 \\
\hline & & 1.3 & 3.4 & 3.1 & 19.0 & 23.1 & 11.5 \\
\hline \multirow[t]{4}{*}{ Florida Staysweet } & 20 & 76.8 & 18.7 & 16.6 & 121.6 & 156.8 & 92.8 \\
\hline & 23 & 73.7 & 12.4 & 14.4 & 110.4 & 137.2 & 51.7 \\
\hline & 26 & 73.1 & 8.1 & 9.8 & 96.8 & 114.7 & 24.4 \\
\hline & & 1.4 & 7.0 & 3.5 & 13.5 & 14.8 & 18.3 \\
\hline \multirow[t]{4}{*}{ FMX 263} & 20 & 75.5 & 22.9 & 25.8 & 266.3 & 315.0 & 76.4 \\
\hline & 23 & 72.6 & 13.7 & 17.7 & 222.0 & 253.4 & 41.7 \\
\hline & 26 & 73.6 & 14.0 & 17.6 & 204.4 & 236.0 & 16.6 \\
\hline & & 1.5 & 3.3 & 4.3 & 45.8 & 47.9 & 10.9 \\
\hline \multirow[t]{4}{*}{ Illini Gold } & 20 & 74.5 & 17.2 & 21.4 & 282.4 & 321.0 & 54.9 \\
\hline & 23 & 72.6 & 10.8 & 14.2 & 235.9 & 261.0 & 46.7 \\
\hline & 26 & 71.6 & 10.3 & 14.0 & 183.0 & 207.3 & 16.2 \\
\hline & & 1.2 & 4.1 & 4.4 & 32.4 & 36.5 & 20.6 \\
\hline \multirow[t]{4}{*}{ Landmark } & 20 & 74.5 & 13.8 & 16.0 & 184.8 & 214.6 & 97.0 \\
\hline & 23 & 72.4 & 18.3 & 22.3 & 237.2 & 277.8 & 40.0 \\
\hline & 26 & 70.9 & 14.1 & 17.6 & 193.6 & 225.3 & 22.8 \\
\hline & & 0.9 & 3.7 & 3.1 & 39.8 & 36.3 & 16.0 \\
\hline \multirow[t]{4}{*}{ Northern Extrasweet } & 20 & 75.0 & 32.7 & 38.1 & 221.4 & 292.2 & 117.9 \\
\hline & 23 & 72.3 & 33.7 & 36.7 & 210.1 & 280.5 & 82.6 \\
\hline & 26 & 70.3 & 30.6 & 33.9 & 208.4 & 272.9 & 32.5 \\
\hline & & 1.1 & 3.4 & 2.6 & 32.8 & 28.7 & 16.1 \\
\hline \multirow[t]{4}{*}{ Pinnacle } & 20 & 75.6 & 35.3 & 37.8 & 237.2 & 310.3 & 154.3 \\
\hline & 23 & 73.8 & 28.0 & 31.0 & 188.2 & 247.2 & 64.4 \\
\hline & 26 & 72.6 & 24.1 & 26.5 & 166.7 & 217.3 & 41.3 \\
\hline & & 1.1 & 5.6 & 4.6 & 62.4 & 60.4 & 36.4 \\
\hline \multirow[t]{4}{*}{ SCH 4006} & 20 & 74.6 & 25.2 & 33.3 & 268.1 & 326.6 & 92.1 \\
\hline & 23 & 73.3 & 23.0 & 26.2 & 242.7 & 291.9 & 62.7 \\
\hline & 26 & 72.0 & 19.9 & 23.5 & 201.2 & 244.6 & 29.3 \\
\hline & & 1.2 & 10.0 & 7.0 & 42.4 & 50.3 & 17.4 \\
\hline
\end{tabular}

Sweet 82' were genetically unique in that the dominant sugar fraction was in the form of hexose (i.e., fructose and glucose) instead of sucrose. At 20 DAP, sucrose concentrations in sh2 hybrids varied from 33 to $371 \mathrm{mg} \cdot \mathrm{g}^{-1}$ dry weight, while total sugar content ranged from 133 to $455 \mathrm{mg} \cdot \mathrm{g}^{-1}$ dry weight. This range of variability among the $s h 2$ hybrids suggests that allelic variation at other loci is profoundly influencing sucrose and total sugar levels in freshly harvested sweet corn.

Ears from the 20 DAP harvest had the highest kernel sucrose and total sugar concentrations (Table 2). Total sugar levels averaged over the $s h 2$ hybrids dropped from $289 \mathrm{mg} \cdot \mathrm{g}^{-1}$ dry weight at 20 DAP to $186 \mathrm{mg} \cdot \mathrm{g}^{-1}$ dry weight at $29 \mathrm{DAP}$, a total loss of $36 \%$ over a 9-day period, or 4\%/day. Substantial variation among the hybrids was observed in the rates of sugar loss with increasing kernel maturity. For example, of two hybrids with comparable sugar concentrations at 20 DAP, the average sugar loss per day was only $2.8 \%$ for 'Northern Extrasweet', but was $5.7 \%$ for 'Illini Gold'. Soberalske and Andrew (1978) have emphasized the importance of considering both the amount of sugar and its rate of change in varietal selection and breeding programs, since both factors influence sweet corn quality.

In 1990, the general response in the various kernel chemical quality components for the six sh2 hybrids at the IRVSF (Table 3) was comparable to results in the 1989 field trial at Urbana, with 
Table 2. Kernel composition for hybrids grown at South Urbana Research Farm (cont.).

\begin{tabular}{|c|c|c|c|c|c|c|c|}
\hline \multirow[b]{2}{*}{ Hybrid } & \multirow[b]{2}{*}{ DAP } & \multirow{2}{*}{$\begin{array}{c}\text { Moisture } \\
(\%)\end{array}$} & Fructose & Glucose & Sucrose & $\begin{array}{c}\text { Total } \\
\text { sugars }\end{array}$ & \multirow{2}{*}{$\begin{array}{c}\text { DMS } \\
\left(\mu \mathrm{g} \cdot \mathrm{g}^{-1}\right)\end{array}$} \\
\hline & & & \multicolumn{3}{|c|}{$\left(\mathrm{mg} \cdot \mathrm{g}^{-1}\right)$} & & \\
\hline \multirow[t]{4}{*}{$\mathrm{SCH} 4415$} & 20 & 73.7 & 34.4 & 37.4 & 282.0 & 353.8 & 54.6 \\
\hline & 23 & 72.1 & 29.7 & 32.9 & 290.5 & 353.1 & 27.4 \\
\hline & 26 & 69.1 & 28.0 & 30.7 & 226.3 & 285.0 & 25.8 \\
\hline & & 1.2 & 7.5 & 8.2 & 68.4 & 74.0 & 16.9 \\
\hline \multirow[t]{4}{*}{ Style Sweet } & 20 & 75.4 & 41.3 & 42.3 & 308.3 & 391.9 & 80.8 \\
\hline & 23 & 74.8 & 48.9 & 50.9 & 289.7 & 389.5 & 58.4 \\
\hline & 26 & 75.3 & 24.9 & 30.1 & 324.9 & 379.9 & 36.8 \\
\hline & & 1.6 & 15.7 & 13.4 & 72.0 & 65.0 & 14.5 \\
\hline \multirow[t]{4}{*}{ Sucro } & 20 & 73.2 & 26.0 & 36.1 & 210.5 & 272.6 & 98.4 \\
\hline & 23 & 72.0 & 39.4 & 47.7 & 209.3 & 296.4 & 50.4 \\
\hline & 26 & 71.5 & 22.8 & 29.2 & 164.6 & 216.6 & 39.1 \\
\hline & & 1.0 & 14.2 & 14.4 & 49.9 & 66.9 & 15.5 \\
\hline \multirow[t]{4}{*}{ Summer Sweet 7210} & 20 & 75.1 & 42.9 & 46.5 & 185.2 & 274.6 & 206.6 \\
\hline & 23 & 72.1 & 23.6 & 32.8 & 170.8 & 227.2 & 109.6 \\
\hline & 26 & 69.5 & 27.0 & 33.6 & 158.1 & 218.8 & 59.9 \\
\hline & & 1.9 & 15.2 & 12.8 & 35.5 & 47.0 & 44.3 \\
\hline \multirow[t]{4}{*}{ Summer Sweet 8000} & 20 & 74.6 & 37.6 & 36.0 & 228.7 & 302.3 & 112.0 \\
\hline & 23 & 74.1 & 29.1 & 29.4 & 195.0 & 253.4 & 72.8 \\
\hline & 26 & 73.6 & 22.4 & 22.4 & 192.7 & 237.6 & 39.4 \\
\hline & & 1.6 & 9.0 & 7.6 & 41.0 & 34.8 & 16.8 \\
\hline \multirow[t]{4}{*}{ Supersweet Jubilee } & 20 & 74.6 & 26.8 & 30.2 & 223.8 & 280.8 & 56.1 \\
\hline & 23 & 73.7 & 23.0 & 27.0 & 207.4 & 257.4 & 33.4 \\
\hline & 26 & 73.6 & 16.7 & 20.4 & 171.8 & 208.9 & 10.6 \\
\hline & & 2.0 & 6.1 & 6.7 & 36.8 & 46.8 & 10.7 \\
\hline \multirow[t]{4}{*}{ Sweet Belle } & 20 & 74.8 & 32.9 & 32.9 & 267.3 & 333.2 & 81.8 \\
\hline & 23 & 73.0 & 30.1 & 30.2 & 218.4 & 278.8 & 47.3 \\
\hline & 26 & 73.0 & 19.8 & 20.0 & 206.3 & 246.2 & 69.8 \\
\hline & & 0.7 & 6.8 & 5.8 & 41.4 & 44.9 & 21.3 \\
\hline \multirow[t]{4}{*}{ Sweetie 70} & 20 & 72.8 & 42.3 & 38.6 & 194.8 & 275.7 & 95.9 \\
\hline & 23 & 71.1 & 35.6 & 33.7 & 174.9 & 244.2 & 66.6 \\
\hline & 26 & 68.2 & 27.5 & 26.3 & 153.9 & 207.6 & 31.0 \\
\hline & & 2.0 & 9.9 & 8.1 & 37.8 & 47.2 & 22.2 \\
\hline \multirow[t]{4}{*}{ Sweetie 76} & 20 & 73.7 & 29.6 & 28.1 & 156.6 & 214.2 & 79.3 \\
\hline & 23 & 73.7 & 19.9 & 19.0 & 151.8 & 190.6 & 66.9 \\
\hline & 26 & 73.3 & 19.3 & 21.1 & 137.2 & 177.7 & 28.4 \\
\hline & & 0.9 & 6.7 & 3.8 & 16.7 & 15.0 & 14.0 \\
\hline \multirow[t]{4}{*}{ Sweetie 82} & 20 & 75.7 & 33.0 & 29.8 & 174.7 & 237.6 & 110.5 \\
\hline & 23 & 74.4 & 28.6 & 27.1 & 151.0 & 206.8 & 77.3 \\
\hline & 26 & 74.1 & 22.5 & 22.3 & 120.9 & 165.7 & 33.0 \\
\hline & & 0.7 & 9.6 & 8.0 & 29.3 & 40.3 & 18.0 \\
\hline \multirow[t]{4}{*}{ Upmost } & 20 & 74.3 & 59.0 & 55.6 & 35.5 & 150.2 & 130.9 \\
\hline & 23 & 71.9 & 63.6 & 62.0 & 29.8 & 155.4 & 70.6 \\
\hline & 26 & 70.0 & 49.6 & 46.1 & 37.8 & 133.5 & 26.2 \\
\hline & & 0.9 & 18.8 & 17.8 & 26.6 & 42.2 & 28.8 \\
\hline
\end{tabular}

kernel moisture content and sugar levels decreasing with increasing harvest maturity. Averaged over the six genotypes, kernel total sugar concentration at 20 DAP was approximately equivalent between the two locations (325 $\mathrm{mg} \cdot \mathrm{g}^{-1}$ at IRVSF vs. $298 \mathrm{mg} \cdot \mathrm{g}^{-1}$ at Urbana). As was observed with yield, several of the hybrids displayed substantial differences in kernel sugar levels, depending on environmental variability. In 'Crisp-N-Sweet 710', kernel total sugar concentrations at 20 and $23 \mathrm{DAP}$ averaged $\approx 32 \%$ higher at Urbana than at the IRVSF, whereas the opposite effect occurred in 'Supersweet Jubilee' and 'Sweetie 82', with $25 \%$ and $72 \%$ more sugars in kernels from the sandy soil site at the IRVSF than at Urbana, respectively.
Significant differences were also found in the amounts of DMS generated from kernel samples among the sh2 hybrids at a given harvest date and over harvest dates for individual hybrids at both locations (Tables 2 and 3). At Urbana, DMS levels at 20 DAP varied from $54.6 \mu \mathrm{g} \cdot \mathrm{g}^{-1}$ dry weight in ' $\mathrm{SCH} 4415$ ' to 206.6 $\mu \mathrm{g} \cdot \mathrm{g}^{-1}$ dry weight in 'Summer Sweet 7210 ', with an average concentration of $94.9 \mu \mathrm{g} \cdot \mathrm{g}^{-1}$ for the 31 hybrids. At the IRVSF, kernel DMS levels at 20 DAP averaged $89.4 \mu \mathrm{g} \cdot \mathrm{g}^{-1}$, ranging from $50.5 \mu \mathrm{g} \cdot \mathrm{g}^{-1}$ in 'FMX 263' to $125.6 \mu \mathrm{g} \cdot \mathrm{g}^{-1}$ in 'Crisp-N-Sweet 710'.

Dimethyl sulfide concentrations in all genotypes decreased with increasing kernel maturity. Mean DMS levels in the $24 s h 2$ hybrids at Urbana dropped 40\% from 20 to 23 DAP, $47 \%$ from 23 
Table 2. Kernel composition for hybrids grown at South Urbana Research Farm (cont.).

\begin{tabular}{|c|c|c|c|c|c|c|c|}
\hline \multirow[b]{2}{*}{ Hybrid } & \multirow[b]{2}{*}{ DAP } & \multirow{2}{*}{$\begin{array}{c}\text { Moisture } \\
(\%)\end{array}$} & Fructose & Glucose & Sucrose & $\begin{array}{c}\text { Total } \\
\text { Sugars }\end{array}$ & \multirow{2}{*}{$\begin{array}{c}\text { DHS } \\
\left(\mu \mathrm{g} \cdot \mathrm{g}^{-1}\right)\end{array}$} \\
\hline & & & \multicolumn{4}{|c|}{$\left(\mathrm{mg} \cdot \mathrm{g}^{-1}\right)$} & \\
\hline \multirow[t]{4}{*}{ Wisc. Natl. Sweet 9000} & 20 & 75.5 & 51.8 & 48.3 & 33.1 & 133.2 & 93.0 \\
\hline & 23 & 74.1 & 48.8 & 40.6 & 15.4 & 104.8 & 24.0 \\
\hline & 26 & 73.8 & 37.4 & 30.7 & 40.5 & 108.7 & 28.4 \\
\hline & & 1.4 & 17.9 & 16.0 & 37.6 & 44.4 & 24.8 \\
\hline \multirow[t]{4}{*}{ Xtra Sweet 82} & 20 & 74.5 & 79.4 & 74.1 & 93.3 & 246.8 & 92.6 \\
\hline & 23 & 72.7 & 70.6 & 61.2 & 46.3 & 178.1 & 56.2 \\
\hline & 26 & 70.4 & 57.5 & 49.0 & 43.5 & 150.0 & 35.1 \\
\hline & & 1.2 & 20.4 & 14.5 & 42.6 & 51.7 & 12.8 \\
\hline \multirow[t]{4}{*}{ Bellringer su1 } & 20 & 69.8 & 29.7 & 30.1 & 41.9 & 101.2 & 82.8 \\
\hline & 23 & 65.9 & 25.9 & 28.7 & 41.4 & 96.0 & 62.2 \\
\hline & 26 & 63.1 & 22.8 & 26.7 & 37.4 & 86.9 & 23.4 \\
\hline & & 1.6 & 2.0 & 1.4 & 8.7 & 7.3 & 18.7 \\
\hline \multirow[t]{4}{*}{ FMX 261 su1 } & 20 & 72.2 & 26.9 & 25.2 & 66.7 & 118.8 & 72.4 \\
\hline & 23 & 68.2 & 28.7 & 28.2 & 67.5 & 114.4 & 43.8 \\
\hline & 26 & 65.4 & 18.2 & 19.6 & 67.8 & 105.6 & 29.9 \\
\hline & & 1.8 & 5.8 & 5.5 & 10.7 & 12.4 & 16.2 \\
\hline \multirow[t]{4}{*}{ Jubilee su1 } & 20 & 71.1 & 34.6 & 32.4 & 57.9 & 124.9 & 57.5 \\
\hline & 23 & 67.3 & 26.9 & 26.8 & 45.8 & 99.4 & 28.7 \\
\hline & 26 & 64.2 & 24.1 & 23.8 & 33.6 & 81.5 & 15.4 \\
\hline & & 2.5 & 5.4 & 5.0 & 16.4 & 19.0 & 9.9 \\
\hline \multirow[t]{4}{*}{ Seneca Horizon su1 } & 20 & 71.9 & 43.6 & 42.1 & 80.1 & 165.7 & 106.8 \\
\hline & 23 & 69.1 & 33.9 & 33.8 & 49.0 & 116.8 & 71.3 \\
\hline & 26 & 65.5 & 24.4 & 25.9 & 56.4 & 106.8 & 42.5 \\
\hline & & 1.1 & 13.2 & 12.2 & 22.8 & 39.9 & 37.7 \\
\hline \multirow[t]{4}{*}{ Style Pack su1 } & 20 & 71.6 & 25.2 & 25.2 & 56.9 & 107.3 & 88.4 \\
\hline & 23 & 68.9 & 19.5 & 20.7 & 55.3 & 95.4 & 54.3 \\
\hline & 26 & 67.0 & 15.5 & 15.8 & 43.2 & 74.4 & 19.4 \\
\hline & & 2.1 & 4.6 & 4.9 & 11.2 & 11.9 & 15.0 \\
\hline \multirow[t]{4}{*}{ Maple Sweet se1 } & 20 & 70.0 & 38.6 & 33.4 & 171.2 & 243.3 & 78.0 \\
\hline & 23 & 68.5 & 32.0 & 31.8 & 133.2 & 196.9 & 42.9 \\
\hline & 26 & 66.7 & 23.6 & 26.6 & 109.3 & 159.5 & 8.6 \\
\hline & & 1.1 & 8.0 & 7.2 & 20.8 & 36.7 & 18.6 \\
\hline \multirow[t]{7}{*}{ Merlin Supersweet se1 } & 20 & 72.2 & 45.2 & 38.7 & 70.3 & 154.1 & 75.6 \\
\hline & 23 & 69.6 & 37.5 & 34.6 & 81.3 & 153.4 & 50.0 \\
\hline & 26 & 67.2 & 35.6 & 34.8 & 68.4 & 138.7 & 29.1 \\
\hline & & 1.5 & 7.1 & 5.3 & 23.5 & 23.2 & 15.7 \\
\hline & 20 & 1.3 & 11.7 & 10.3 & 40.1 & 45.8 & 28.2 \\
\hline & 23 & 1.1 & 9.9 & 8.7 & 39.2 & 44.2 & 18.6 \\
\hline & 26 & 1.2 & 8.4 & 7.6 & 29.6 & 33.7 & 12.7 \\
\hline
\end{tabular}

$\mathrm{z}_{\text {sh2 }}$ endosperm mutation unless otherwise indicated.

yTo reduce table size, data at 29 DAP were not presented since concentrations of kernel chemical composition mostly leveled off by this harvest date. LSD between harvest maturities for each hybrid at $P=0.5$ is presented on the fourth line of each entry.

${ }^{\mathrm{x}}$ LSD between hybrid means at each harvest maturity at $P=0.05$.

to 26 DAP, and another $40 \%$ from 26 to 29 DAP. Dimethyl sulfide levels at 29 DAP were only $19 \%$ of those assayed at 20 DAP kernel samples, an average reduction of $9 \% /$ day.

Comparison of DMS concentrations between the two locations revealed that, on the average, 20 DAP kernels from Urbana generated $37 \%$ more of the compound than samples from IRVSF. This difference could be attributed to differences in rates of kernel maturation and/or soil environment associated with different locations. In contrast to kernel sugar content, the relative ranking of genotypes for DMS content over planting locations did not vary dramatically.

Sources of variation in yield and yield component evaluations and the chemical composition of quality. Following ANOVA, the total variability (based on the sum of squares) for each trait was partitioned into component sources of variation due to genotype, environment, genotype by environment interaction, and an error term. The relative contribution of each source of variation to the total variability of a trait is presented in Table 4 as percentages.

With the exception of kernel depth, main effects for genotype contributed to the major portion of the total variation in the various yield and yield component parameters. Significant amounts of variation in ear weight, potential yield, and kernel yield was attributable to genotype by environmental interactions. Kernel maturity, as measured by moisture content, was also primarily under the control of genotypic variation. With nearly $80 \%$ of the variability associated with genotypic differences, the genes con- 
Table 3. Kernel composition for hybrids grown at the Illinois River Valley Sand Field.

\begin{tabular}{|c|c|c|c|c|c|c|c|}
\hline \multirow[b]{2}{*}{ Hybrid $^{z}$} & \multirow[b]{2}{*}{ DAP $^{y}$} & \multirow{2}{*}{$\begin{array}{c}\text { Moisture } \\
(\%)\end{array}$} & Fructose & Glucose & Sucrose & $\begin{array}{c}\text { Total } \\
\text { sugars }\end{array}$ & \multirow{2}{*}{$\begin{array}{c}\text { DMS } \\
\left(\mu \mathrm{g} \cdot \mathrm{g}^{-1}\right)\end{array}$} \\
\hline & & & \multicolumn{4}{|c|}{$\left(\mathrm{mg} \cdot \mathrm{g}^{-1}\right)$} & \\
\hline \multirow[t]{4}{*}{ Crisp-N-Sweet 710} & 20 & 75.9 & 35.1 & 36.4 & 217.7 & 289.3 & 125.6 \\
\hline & 23 & 74.0 & 28.5 & 29.3 & 238.1 & 296.0 & 91.4 \\
\hline & 26 & 73.7 & 27.6 & 28.9 & 215.8 & 272.3 & 66.8 \\
\hline & & 1.5 & 5.1 & 4.5 & 15.8 & 12.6 & 18.6 \\
\hline \multirow[t]{4}{*}{ FMX 263} & 20 & 76.4 & 29.4 & 31.5 & 274.2 & 335.0 & 50.5 \\
\hline & 23 & 74.8 & 25.0 & 25.5 & 279.5 & 329.9 & 42.0 \\
\hline & 26 & 74.7 & 20.3 & 21.7 & 253.3 & 295.3 & 37.0 \\
\hline & & 0.8 & 2.8 & 3.0 & 15.8 & 18.5 & 12.0 \\
\hline \multirow[t]{4}{*}{ Summer Sweet 7210} & 20 & 76.9 & 23.0 & 25.4 & 216.3 & 264.6 & 122.1 \\
\hline & 23 & 75.3 & 20.3 & 22.4 & 218.3 & 261.0 & 79.5 \\
\hline & 26 & 74.4 & 16.7 & 18.7 & 196.2 & 231.6 & 50.4 \\
\hline & & 0.6 & 3.4 & 3.2 & 14.6 & 17.9 & 21.8 \\
\hline \multirow[t]{4}{*}{ Supersweet Jubilee } & 20 & 74.9 & 30.6 & 30.5 & 297.3 & 358.5 & 55.5 \\
\hline & 23 & 73.1 & 21.2 & 24.8 & 270.4 & 316.3 & 29.4 \\
\hline & 26 & 74.3 & 17.2 & 21.2 & 269.5 & 307.9 & 27.5 \\
\hline & & 1.4 & 4.8 & 4.1 & 13.9 & 20.6 & 8.6 \\
\hline \multirow[t]{4}{*}{ Sweetie 70} & 20 & 74.6 & 28.5 & 28.0 & 251.8 & 308.2 & 65.0 \\
\hline & 23 & 73.0 & 26.0 & 24.6 & 229.6 & 280.1 & 44.4 \\
\hline & 26 & 71.6 & 17.7 & 18.6 & 225.0 & 261.3 & 31.4 \\
\hline & & 1.3 & 2.4 & 2.2 & 12.8 & 12.5 & 11.2 \\
\hline \multirow[t]{7}{*}{ Sweetie 82} & 20 & 76.1 & 36.1 & 34.1 & 319.6 & 389.8 & 117.4 \\
\hline & 23 & 76.0 & 21.6 & 23.9 & 327.5 & 373.0 & 74.4 \\
\hline & 26 & 75.7 & 18.0 & 22.1 & 315.5 & 355.6 & 55.6 \\
\hline & & 0.6 & 5.1 & 5.1 & 41.4 & 42.6 & 14.3 \\
\hline & 20 & 1.0 & 5.0 & 4.6 & 20.1 & 22.0 & 17.6 \\
\hline & 23 & 1.1 & 3.5 & 3.2 & 16.7 & 18.8 & 13.2 \\
\hline & 26 & 1.2 & 3.4 & 3.3 & 25.7 & 26.7 & 13.2 \\
\hline
\end{tabular}

${ }_{\mathrm{z}} \mathrm{sh2}$ endosperm mutation unless otherwise indicated.

${ }^{\mathrm{y}} \mathrm{LSD}$ between harvest maturities for each hybrid at $P=0.05$ is presented on the fourth line of each hybrid entry.

${ }^{\mathrm{x}} \mathrm{LSD}$ between hybrid means at each harvest maturity at $P=0.05$ is presented in the bottom three lines of the table.

trolling kernel DMS variation in the hybrids appear to have similar phenotypic expression in both planting locations. In contrast, fructose, glucose, sucrose, and total sugar concentrations, which determine kernel sweetness, were primarily influenced by the interaction between the genotype and the environment.

Comparative performance of sh2 and sul hybrids. Mean performance of sh 2 hybrids for yield and yield component parameters and kernel quality characteristics was in all cases equal or better than that of hybrids homozygous for the sul mutation (Table 5). Ear uniformity of $s h 2$ hybrids was very close to being significantly superior $(P=0.052)$ to the sul hybrids. On the basis of yield and yield component evaluations in this study, the perception that $s h 2$ hybrids give lower yields than sul is unfounded, since ear and cut kernel yield were about equal in both endosperm types.

As expected from the action of sh2 gene (Laughnan, 1953), kernel moisture content, sucrose level, and total sugar concentration were significantly greater in $s h 2$ than in sul hybrids. With mean DMS levels of 99.1 and $81.6 \mu \mathrm{g} \cdot \mathrm{g}^{-1}$ for $s h 2$ and sul hybrids, respectively, the type of endosperm mutation did not significantly influence the concentration of kernel DMS.

Correlation of yield, yield components, and kernel quality characteristics. To ascertain if key traits display positive or negative associations, correlation coefficients were computed among yield and yield component variables at 23 DAP, and between kernel chemical components at 20 and 23 DAP for the 24 $s h 2$ hybrids harvested at South Urbana Research Farm (Table 6). All of the yield and yield component parameters were correlated $(P$
$<0.01)$. This response was expected since ear weight, ear length, and kernel depth were used to calculate potential and kernel yield. Similarly, the association between kernel moisture and yield also was expected, since water is the largest single component determining kernel weight and consequently contributes to increased yield. Ear uniformity and potential yield of the ear did not correlate with kernel sucrose, total sugar, and DMS concentrations, suggesting that these traits are controlled by genes operating independently. Kernel yield, while unrelated to sucrose level and DMS concentration, was negatively correlated with total sugar concentration in the kernel, although the value of $r$ was low $(r=-0.22)$. A negative correlation was observed between fructose and sucrose, but no relationship between glucose and sucrose was found.

The selection of hybrids for commercial production or for sources of favorable alleles in a breeding program for sweet corn improvement will depend on the consistency of a cultivar's performance over environments. Our replicated study at two locations with six of the $s h 2$ hybrids was conducted to provide information concerning the effect of environmental factors on the performance of these hybrids. Not only do the two planting sites have distinctly different soil types, but they were used in separate years to maximize the environmental effects on yield-related traits and on kernel chemical composition. Partitioning the variability in yield and kernel chemical components showed that genetic differences among the hybrids generally are the primary source of variation affecting yield, yield component characteristics, and kernel quality, although there were significant environmental effects, particu- 
Table 4. Percentage of variability explained by the model and by each source of variation in the model for yield and yield component evaluations at 23 DAP and kernel chemical composition at 20 DAP for six sh2 hybrids harvested at South Urbana Research Farm and the Illinois River Valley Sand Field station. ${ }^{\mathrm{z}}$

\begin{tabular}{|c|c|c|c|c|c|}
\hline Variable & Model $^{y}$ & Genotype $^{\mathrm{x}}$ & Environment & $\begin{array}{c}\text { Genotype } \times \\
\text { environment }\end{array}$ & $\begin{array}{l}\text { Error } \\
\text { term }^{\mathrm{w}}\end{array}$ \\
\hline \multicolumn{6}{|c|}{ Yield and yield components at 23 DAP } \\
\hline Ear uniformity & 62.3 & $\begin{array}{c}70.0 \\
(0.0002)\end{array}$ & $\begin{array}{c}11.4 \\
(0.0455)\end{array}$ & $\begin{array}{c}8.2 \\
(0.5518)\end{array}$ & 10.3 \\
\hline Ear weight & 78.6 & $\begin{array}{c}60.7 \\
(<0.0001)\end{array}$ & $\begin{array}{c}2.9 \\
(0.2048)\end{array}$ & $\begin{array}{c}26.0 \\
(0.0008)\end{array}$ & 10.3 \\
\hline Ear length & 62.2 & $\begin{array}{c}71.2 \\
(0.0002)\end{array}$ & $\begin{array}{c}4.2 \\
(0.2562)\end{array}$ & $\begin{array}{c}11.1 \\
(0.3869)\end{array}$ & 13.5 \\
\hline Kernel depth & 94.3 & $\begin{array}{c}20.4 \\
(<0.0001)\end{array}$ & $\begin{array}{c}77.1 \\
(<0.0001)\end{array}$ & $\begin{array}{c}1.3 \\
(0.2891)\end{array}$ & 1.3 \\
\hline Potential yield & 78.6 & $\begin{array}{c}60.8 \\
(<0.0001)\end{array}$ & $\begin{array}{c}2.9 \\
(0.2053)\end{array}$ & $\begin{array}{c}26.0 \\
(0.0008)\end{array}$ & 10.3 \\
\hline Kernel yield & 84.9 & $\begin{array}{c}54.4 \\
(<0.0001)\end{array}$ & $\begin{array}{c}26.7 \\
(0.0091)\end{array}$ & $\begin{array}{c}10.4 \\
(0.0134)\end{array}$ & 8.5 \\
\hline \multicolumn{6}{|c|}{ Kernel chemical composition at 20 DAP } \\
\hline Moisture content & 70.1 & $\begin{array}{c}55.9 \\
(<0.0001)\end{array}$ & $\begin{array}{c}12.8 \\
(0.1461)\end{array}$ & $\begin{array}{c}13.0 \\
(0.1369)\end{array}$ & 18.1 \\
\hline Fructose & 67.2 & $\begin{array}{c}33.8 \\
(0.0056)\end{array}$ & $\begin{array}{c}10.0 \\
(0.0595)\end{array}$ & $\begin{array}{c}47.9 \\
(0.0007)\end{array}$ & 8.3 \\
\hline Glucose & 76.6 & $\begin{array}{c}37.5 \\
(<0.0001)\end{array}$ & $\begin{array}{c}14.5 \\
(0.0170)\end{array}$ & $\begin{array}{c}43.6 \\
(<0.0001)\end{array}$ & 4.4 \\
\hline Sucrose & 85.0 & $\begin{array}{c}26.7 \\
(<0.0001)\end{array}$ & $\begin{array}{c}13.4 \\
(0.0034)\end{array}$ & $\begin{array}{c}57.4 \\
(<0.0001)\end{array}$ & 2.6 \\
\hline Total sugar & 83.0 & $\begin{array}{c}24.0 \\
(0.0002)\end{array}$ & $\begin{array}{c}6.4 \\
(0.0178)\end{array}$ & $\begin{array}{c}65.3 \\
(<0.0001)\end{array}$ & 4.3 \\
\hline DMS & 89.2 & $\begin{array}{c}77.0 \\
(<0.0001)\end{array}$ & $\begin{array}{c}13.4 \\
(0.0034)\end{array}$ & $\begin{array}{c}8.6 \\
(0.0045)\end{array}$ & 0.9 \\
\hline
\end{tabular}

$\overline{\mathrm{z}}$ Values were calculated by dividing the sum of squares of the factor involved by the total sum of squares in the model expressed as percentages.

${ }^{\mathrm{y}}$ Consisted of genotype, environment, and genotype $\times$ environment variability.

${ }^{\mathrm{x}}$ Numbers in parenthesis are $P$ values.

${ }^{\mathrm{w}}$ Composed of rep and rep $\times$ environment variability.

Table 5. Mean comparisons between sh2 and sul hybrids for yield and yield component evaluations harvested at 23 DAP and kernel chemical composition harvested at 20 DAP at South Urbana Research Farm.

\begin{tabular}{|c|c|c|c|}
\hline \multirow[b]{2}{*}{ Variable } & \multicolumn{2}{|c|}{ Mean performance } & \multirow{2}{*}{$\begin{array}{c}P \\
\text { value }^{\mathrm{z}}\end{array}$} \\
\hline & sh2 hybrid & sul hybrid & \\
\hline \multicolumn{4}{|c|}{ Yield and yield components at 23 DAP } \\
\hline Ear uniformity, 0 to 4 & 2.30 & 2.20 & 0.0520 \\
\hline Ear weight, $g$ & 225 & 206 & 0.3420 \\
\hline Ear length, cm & 19.9 & 19.2 & 0.2711 \\
\hline Kernel depth, cm & 0.79 & 0.78 & 0.2923 \\
\hline Potential yield, MT $\cdot \mathrm{ha}^{-1}$ & 9.99 & 9.15 & 0.3022 \\
\hline Kernel yield, MT $\cdot \mathrm{ha}^{-1}$ & 5.72 & 4.99 & 0.5688 \\
\hline \multicolumn{4}{|c|}{ Kernel chemical composition at 20 DAP } \\
\hline Moisture content, \% & 74.6 & 71.3 & 0.0025 \\
\hline Fructose, $\mathrm{mg} \cdot \mathrm{g}^{-1}$ & 34.3 & 31.9 & 0.3292 \\
\hline Glucose, $\mathrm{mg} \cdot \mathrm{g}^{-1}$ & 35.8 & 31.0 & 0.1069 \\
\hline Sucrose, $\mathrm{mg} \cdot \mathrm{g}^{-1}$ & 210 & 60.7 & $<0.0001$ \\
\hline Total sugar, $\mathrm{mg} \cdot \mathrm{g}^{-1}$ & 280 & 124 & $<0.0001$ \\
\hline $\mathrm{DMS}, \mu \mathrm{g} \cdot \mathrm{g}^{-1}$ & 99.1 & 81.6 & 0.0540 \\
\hline
\end{tabular}

${ }^{\mathrm{z}}$ Computed using Student's $t$ test. larly on kernel sugar concentrations.

Of all the chemical parameters of sweet corn eating quality measured in this study, DMS displayed the greatest reduction with kernel age. This rapid loss of aromatic quality is of particular concern for the sweet corn processing industry. Apparently, Smethylmethionine, which is a precursor of DMS (Bills and Keenan, 1968; Wong et al., 1991), is undergoing active conversion to other metabolic products during this period. This stage of kernel development also corresponds to a period when kernel protein metabolism is shifting from the synthesis of water soluble enzymes to the accumulation of zein storage proteins (Bjarnason and Vasal, 1992).

Under conditions of similar emergence and stand uniformity, these data showed that $s h 2$ hybrids display superior quality with yields comparable to traditional sul cultivars. The sh2 hybrids were in all cases equal, or better than that of the sul hybrids for yield, yield component, and kernel quality parameters. Since both ear and kernel yields were not significantly different for both endosperm types, the perception that $s h 2$ hybrids give lower yield than $s u l$ is unfounded. The substantial variability among the $s h 2$ hybrids, with a major proportion of the variation attributable to genotypic differences and the lack of strong negative associations between yield and important chemical components of kernel quality, suggests that it should be feasible to develop sh2 hybrids with satisfactory yield potential and improved eating quality for specialized sweet corn markets. 
Table 6. Pearson's correlation coefficients for yield and yield component evaluations at 23 DAP and kernel chemical composition at 20 and 23 DAP for the $24 \operatorname{sh} 2$ hybrids harvested at South Urbana Research Farm.

\begin{tabular}{|c|c|c|c|c|c|c|c|c|c|c|c|c|}
\hline Variable & DAP & EW & EL & $\mathrm{KD}$ & PY & KY & $\mathrm{MC}$ & FR & GL & SU & $\mathrm{TS}$ & DMS \\
\hline \multicolumn{13}{|c|}{ Yield and yield components } \\
\hline Ear uniformity & 23 & $\begin{array}{c}0.60 \\
(<0.0001)\end{array}$ & $\begin{array}{c}0.32 \\
(0.0017)\end{array}$ & $\begin{array}{c}0.43 \\
(<0.0001)\end{array}$ & $\begin{array}{c}0.60 \\
(<0.0001)\end{array}$ & $\begin{array}{c}0.53 \\
(<0.0001)\end{array}$ & NS & $\begin{array}{c}-0.27 \\
(0.0086)\end{array}$ & $\begin{array}{c}-0.28 \\
(0.0049)\end{array}$ & NS & NS & NS \\
\hline Ear weight (EW) & 23 & & $\begin{array}{c}0.47 \\
(<0.0001)\end{array}$ & $\begin{array}{c}0.63 \\
(<0.0001)\end{array}$ & $\begin{array}{c}0.99 \\
(<0.0001)\end{array}$ & $\begin{array}{c}0.85 \\
(<0.0001)\end{array}$ & $\begin{array}{c}0.23 \\
0.0242)\end{array}$ & NS & NS & NS & NS & NS \\
\hline Ear length (EL) & 23 & & & $\begin{array}{c}0.21 \\
(0.0441)\end{array}$ & $\begin{array}{c}0.47 \\
(<0.0001)\end{array}$ & $\begin{array}{c}0.36 \\
(0.0004)\end{array}$ & NS & NS & NS & NS & NS & NS \\
\hline Kernel depth (KD) & 23 & & & & $\begin{array}{c}0.63 \\
(<0.0001)\end{array}$ & $\begin{array}{c}0.70 \\
(<0.0001)\end{array}$ & NS & NS & NS & NS & NS & NS \\
\hline Potential yield (PY) & 23 & & & & & $\begin{array}{c}0.85 \\
(<0.0001)\end{array}$ & $\begin{array}{c}0.23 \\
0.0236)\end{array}$ & NS & NS & NS & NS & NS \\
\hline Kernel Yield (KY) & 23 & & & & & ( & $\begin{array}{c}0.24 \\
0.0176)\end{array}$ & NS & NS & NS & $\begin{array}{c}-0.22 \\
0.0332\end{array}$ & NS \\
\hline \multicolumn{13}{|c|}{ Kernel chemical composition } \\
\hline Moisture content (MC) & 20 & & & & & & & NS & NS & NS & NS & $\begin{array}{c}0.32 \\
(0.0014)\end{array}$ \\
\hline & 23 & & & & & & & NS & NS & NS & NS & NS \\
\hline \multirow[t]{2}{*}{ Fructose (FR) } & 20 & & & & & & & & $\begin{array}{c}0.96 \\
(<0.0001)\end{array}$ & $\begin{array}{c}-0.28 \\
(0.0052)\end{array}$ & NS & $\begin{array}{c}0.25 \\
(0.0155)\end{array}$ \\
\hline & 23 & & & & & & & & $\begin{array}{c}0.96 \\
(<0.0001)\end{array}$ & $\begin{array}{c}-0.30 \\
(0.0026)\end{array}$ & NS & $\mathrm{NS}$ \\
\hline \multirow[t]{2}{*}{ Glucose (GL) } & 20 & & & & & & & & & NS & NS & $\begin{array}{c}0.30 \\
(0.0032)\end{array}$ \\
\hline & 23 & & & & & & & & & NS & NS & NS \\
\hline \multirow[t]{2}{*}{ Sucrose (SU) } & 20 & & & & & & & & & & $\begin{array}{c}0.94 \\
(<0.0001)\end{array}$ & NS \\
\hline & 23 & & & & & & & & & & $\begin{array}{c}0.93 \\
(<0.0001)\end{array}$ & NS \\
\hline \multirow[t]{2}{*}{ Total sugar (TS) } & 20 & & & & & & & & & & & NS \\
\hline & 23 & & & & & & & & & & & NS \\
\hline
\end{tabular}

${ }^{\mathrm{z}}$ Numbers in parentheses are $P$ values.

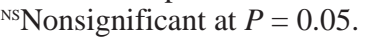

\section{Literature Cited}

Bailey, D.M. and R.M. Bailey. 1938. The relation of the pericarp to tenderness in sweet corn. Proc. Amer. Soc. Hort. Sci. 36:555-559.

Bills, D.D. and T.W. Keenan. 1968. Dimethyl sulfide and its precursor in sweet corn. J. Agr. Food Chem. 16:643-645.

Bjarnason, M. and S.K. Vasal. 1992. Breeding of quality protein maize (QMP). Plant Breeding Rev. 9:181-216.

Breeden, D.C. and J.A. Juvik. 1992. An extraction method for the determination of dimethyl sulfide in cooked corn. J. Food Comp. Anal. 5:134-138.

Carey, E.E., D.B. Dickinson, and A.M. Rhodes. 1984. Sugar characteristics of sweet corn populations from a sugary enhancer breeding program. Euphytica 33:609-622.

Cobb, B.G. and L.C. Hannah. 1981. The metabolism of sugars in maize endosperms. Plant Physiol. 67:107.

Creech, R.G. 1965. Genetic control of carbohydrate synthesis in maize endosperm. Genetics 52:1175-1186.

Culpepper, C.W. and C.A. Magoon. 1927. A study of the factors determining quality in sweet corn. J. Agr. Res. 34:413-433.

Dignan, D.M. and R.C. Wiley. 1976. DMS level in the aroma of cooked frozen sweet corn as affected by cultivar, maturity, blanching, and packaging. J. Food Sci. 41:346-348.

Evensen, K.B. and C.D. Boyer. 1986. Carbohydrate composition of sensory quality of fresh and stored sweet corn. J. Amer. Soc. Hort. Sci. 111:734-738.

Flora, L.F. and R.C. Wiley. 1974. Sweet corn aroma, chemical components, and relative importance in the overall flavor response. J. Food Sci. 39: 770-773.

Garwood, D.L., F.J. McArdle, S.F. Vanderslice, and J.C. Shannon. 1976. Postharvest carbohydrate transformation and processed quality of high sugar maize genotypes. J. Amer. Soc. Hort. Sci. 101:400-404.

Juvik, J.A. and D.R. LaBonte. 1988. Single-kernel analysis for the presence of the sugary enhancer (se) gene in sweet corn. HortScience 23:384-386.

Laughnan, J.R. 1953. The effect of the $s h 2$ factor on carbohydrate reserves in the mature endosperm of maize. Genetics 38:485-499.

Marshall, S.W. 1987. Sweet corn, p. 431-445. In: S.A. Watson and P.E. Ramstad (eds.). Corn: Chemistry and technology. Amer. Assoc. Cereal Chem. Inc., St. Paul, Minn.

Reyes, F.G.R., G.W. Varseveld, and M.C. Kuhn. 1982. Sugar composition and flavour quality of high sugar (shrunken) and normal sweet corn. J. Food Sci. 47:753-755.

Showalter, R.K. and L.W. Miller. 1962. Consumer preference for high-sugar sweet corn varieties. Proc. Fla. State Hort. Soc. 75:278-280.

Soberalske, R.M. and R.H. Andrew. 1978. Gene effects on kernel moisture and sugars of near-isogeneic lines of sweet corn. Crop Sci. 18:743-746.

Steel, R.G.D. and J.H. Torrie. 1960. Principles and procedures of statistics. McGraw-Hill Book Co., New York.

Wann, E.V., G.B. Brown, and W.A. Hills. 1971. Genetic modification of sweet corn quality. J. Amer. Soc. Hort. Sci. 96:441-444.

Wiley, R.C. 1985. Sweet corn aroma: Studies of its chemical components and influence on flavor, p. 349-366. In: H. E. Pattee (ed.). Evaluation of quality of fruits and vegetables. AVI Publishing, Westport, Conn.

Williams, M.P. and P.E. Nelson. 1973. Effects of hybrids and processing on the dimethyl sulfide potential of sweet corn. J. Food Sci. 38:1136-1138.

Wong, A.D., J.A. Juvik, J.M. Swiader, and J.A. Grunau. 1991. Levels of dimethyl sulfide and its precursor in sweet corn as influenced by genotype and harvest maturity. HortScience 26:776. (Abstr.) 